\title{
Driving electromechanically assisted Gait Trainer for people with stroke
}

\author{
Marco Iosa, PhD; ${ }^{1 *}$ Giovanni Morone, MD; ${ }^{1}$ Maura Bragoni, MD, PhD; ${ }^{2}$ Domenico De Angelis, MD; ${ }^{2}$ Vincenzo \\ Venturiero, MD, PhD $;^{2}$ Paola Coiro, $\mathrm{MD} ;{ }^{2}$ Luca Pratesi, $\mathrm{MD} ;{ }^{2}$ Stefano Paolucci, $\mathrm{MD}^{1-2}$ \\ ${ }^{1}$ Movement and Brain Laboratory, Fondazione Santa Lucia, Istituto di Ricerca e Cura a Carattere Scientifico (IRCCS) \\ [Santa Lucia Foundation, Scientific Institute for Research Hospitalization and Health Care], Rome, Italy; ${ }^{2}$ Unità \\ Operativa F [Operative Unit F], Fondazione Santa Lucia, IRCCS, Rome, Italy
}

\begin{abstract}
Electromechanically assisted gait training is a promising task-oriented approach for gait restoration, especially for people with subacute stroke. However, few guidelines are available for selecting the parameter values of the electromechanical Gait Trainer (GT) (Reha-Stim; Berlin, Germany) and none is tailored to a patient's motor capacity. We assessed 342 GT sessions performed by 20 people with stroke who were stratified by Functional Ambulatory Category. In the first GT session of all patients, the body-weight support (BWS) required was higher than that reported in the literature. In further sessions, we noted a slow reduction of BWS and a fast increment of walking speed for the most-affected patients. Inverse trends were observed for the less-affected patients. In all the patients, the heart rate increment was about 20 beats per minute, even for sessions in which the number of strides performed was up to 500. In addition, the effective BWS measured during GT sessions was different from that initially selected by the physiotherapist. This difference depended mainly on the position of the GT platforms during selection. Finally, harness acceleration in the anteroposterior direction proved to be higher in patients with stroke than in nondisabled subjects. Our findings are an initial step toward scientifically selecting parameters in electromechanically assisted gait training.
\end{abstract}

Key words: accelerometry, ambulation recovery, assessment, body-weight support, gait training, heart rate, parameter selection, rehabilitation, stroke, walking speed.

\section{INTRODUCTION}

Over the last decade, devices for electromechanically assisted gait training have been developed that allow nonambulatory patients to perform intensive, task-oriented exercise [1-2]. The effectiveness of these devices has been investigated in patients with subacute [3-5] and chronic [6-7] stroke, but their overall efficacy has been questioned [8-9]. The absence of a general agreement may be due to differences among patients enrolled in these studies, as well as the lack of a unified approach to integrating these devices into rehabilitation programs [10]. The effectiveness of these devices may therefore

\footnotetext{
Abbreviations: $\triangle \mathrm{HR}=$ heart rate increment, $6 \mathrm{MWT}=6$-minute walk test, ANOVA = analysis of variance, AP = anteroposterior, bpm = beats per minute, BWS = body-weight support, $\mathrm{BWS}_{\mathrm{e}}=$ effective BWS, $\mathrm{BWS}_{\mathrm{S}}=\mathrm{BWS}$ in a static posture, $\mathrm{CC}=$ craniocaudal, FAC $=$ Functional Ambulatory Category, GT = Gait Trainer, HR = heart rate, LL = laterolateral, RMS = rootmean-square, $\mathrm{SD}=$ standard deviation, $\mathrm{SL}=$ step length, $\mathrm{WS}=$ walking speed, $\mathrm{WS}_{\mathrm{e}}=$ effective $\mathrm{WS}, \mathrm{WS}_{\text {mean }}=$ mean $\mathrm{WS}$ during entire GT session, $\mathrm{WS}_{\mathrm{S}}=$ selected WS.

*Address all correspondence to Marco Iosa, PhD; Fondazione Santa Lucia-IRCCS, Via Ardeatina 306, 00179 Rome, Italy; +39-06-51-50-1005; fax: +39-06-51-50-1004.

Email: m.iosa@hsantalucia.it

DOI:10.1682/JRRD.2010.04.0069
} 
depend heavily on the ability of the rehabilitation team to most effectively tailor the selection of their parameters to each patient.

Information about the criteria used to select parameters for these devices, including the reasons for choosing the amount of body-weight support (BWS) or imposed walking speeds (WSs), is not usually stated in the literature or user manuals. Currently, therefore, training protocols vary widely among clinical trials. Low percentages of BWS and fast WSs may activate the relevant weightbearing muscles and improve locomotor efficiency [1112] but can overstrain patients [5]. Despite every physiotherapist's concern for selecting the maximum parameter values that each patient can achieve without discomfort, clear guidelines and/or scientific analyses of these values are still lacking, as well as knowledge of their interrelationships and their effects on over-ground walking performance. This absence of guidelines may explain the lack of general agreement about the effectiveness of robotic devices for gait recovery $[10,13]$, the absence of a well-defined optimal training plan for each level of patients' walking dependency [13], and the skepticism sometimes expressed by physicians and physiotherapists toward the use of robotic aids in rehabilitation [14]. Deeper knowledge may increase awareness of the potential of electromechanical-assisted walking training in rehabilitation.

The most common electromechanically assisted gait devices for gait restoration are a treadmill with BWS [15], the Lokomat ${ }^{\circledR}$ (Hocoma, AG; Volketswil, Switzerland) [16], and the Gait Trainer ${ }^{\circledR}$ (GT) (Reha-Stim; Berlin, Germany) [2]. On treadmills, only the percentage of BWS and the WS can be selected. The Lokomat has many more options; the rehabilitation team can even decide the proper joint kinematics. The GT lies between these two extremes (Figure 1). It includes a system for BWS and a controller of endpoint feet trajectories. During a GT session, each of the patient's feet is fixed by straps to a plate moved by the GT engine, which simulates swing (40\%) and stance (60\%) gait phases, by means of a crank and rocker system that includes a planetary gear system [1-2]. The result is a gait-like movement, in which the values of the spatiotemporal parameters are selected by the physiotherapist and imposed on the patients by the device [1-2]. Joint kinetics and kinematics are not imposed but can be influenced by the selection of the values discussed earlier.

During the initial preparation phase, the physiotherapist (two physiotherapists are often required for the most severely affected patients) has to harness the patient and help him to rise on the GT platforms, previously placed in a proper position. Then the physiotherapist should select the following parameters: the needed BWS, the step length (SL), and the WS.

BWS is the essential ingredient, the conditio sine qua non, for nonambulatory patients to practice the exercises intensively and safely. BWS permits a greater number of steps within a training session than conventional therapy, in which body weight is manually supported by one or two physiotherapists and/or a walker $[3,13]$. The physiotherapist initially selects the BWS value in a static posture $\left(\mathrm{BWS}_{\mathrm{s}}\right)$. However, a common standing posture with both lower limbs extended is impossible on the GT, because when one plate is down (simulating the stance phase) the other is up (simulating the swing phase). Furthermore, the effective BWS $\left(\mathrm{BWS}_{\mathrm{e}}\right.$ ) varies during the session, depending on the patient's capacity to sustain his/her own unsupported percentage of weight $[5,17]$. Despite these features, all previous studies on the GT have reported $\mathrm{BWS}_{\mathrm{s}}$ only, without describing the position at which it was selected. Moreover, these studies have tacitly assumed that $\mathrm{BWS}_{\mathrm{S}}$ is a reference value around which the $\mathrm{BWS}_{\mathrm{e}}$ falls during the GT session. However, whether this implicit assumption is true is still not proven.

Evidence is emerging that the prescription of certain parameters in treadmill training with BWS can affect treatment outcome in people with hemiparesis due to stroke. This evidence has motivated investigations of the scientific basis for the proper selection of the device parameters [18]. In this study, we have retrospectively documented the selection of GT parameters based on clinical judgment during almost 400 sessions. We have also analyzed the relationships among selected values, measured quantities, and clinically observed a patient's locomotor capacity. This analysis is in accordance with similar previous investigations on the effects of BWS, WS, and support system stiffness in treadmill training [18]. Our aim was to contribute to the development of a scientific rationale useful in the selection of GT parameters.

\section{METHODS}

We used three different experimental protocols: (1) a retrospective study of 20 patients, each of whom was included in a training program of 20 GT sessions; (2) kinematics (acceleration) and kinetics (force) measured 


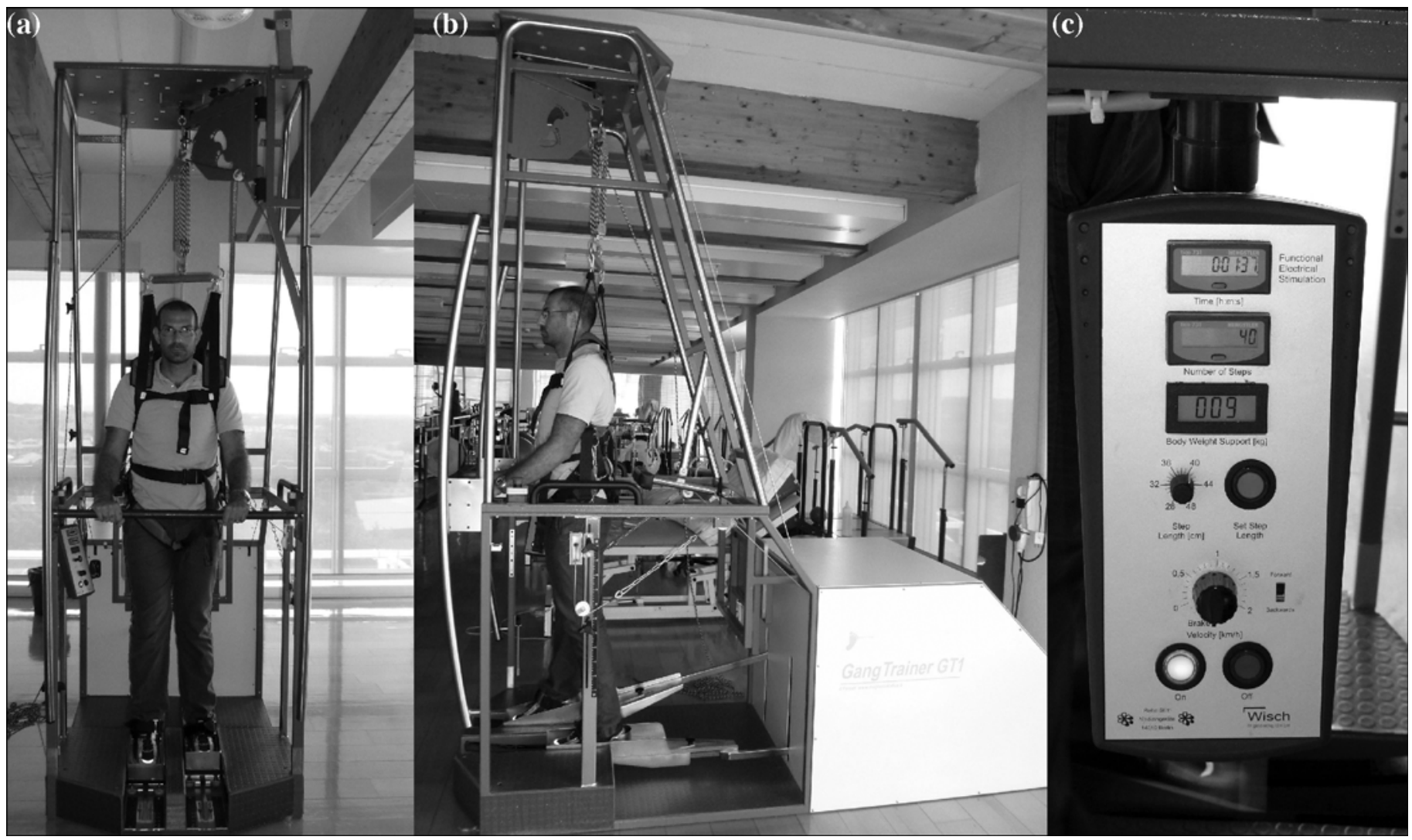

Figure 1.

Gait Trainer (GT): (a) Frontal and (b) lateral views of nondisabled subject performing GT session, and (c) GT controller showing number of strides performed (not number of steps, as incorrectly shown on GT controller) and effective body-weight support measured by GT dynamometer. At bottom are step length selector and walking speed selector (which is actually cadence selector).

during 6 brief GT sessions performed by 8 nondisabled patients each, with BWS measured in 3 different initial positions; and (3) the same kinematic and kinetic parameters measured during GT sessions performed by 7 patients. The inclusion criteria for patients were hemiparesis in the subacute phase with significant gait deficit (Functional Ambulatory Category [FAC] score $\leq 3$ ) due to first-ever stroke with lesion confirmed by neuroimaging. Exclusion criteria included the presence of subarachnoid hemorrhage, sequelae of previous cerebrovascular accidents and/or other chronic disabling pathologies, orthopedic injury that may impair locomotion, important neglect, or a high level of spasticity or cognitive impairment.

Particular attention was paid to BWS, WS, and harness accelerations, similar to the review by Chen and Patten of treadmill training [18]. The selected WS $\left(\mathrm{WS}_{\mathrm{s}}\right)$ should be equal to the effective WS $\left(\mathrm{WS}_{\mathrm{e}}\right)$. However, the WS selector of the GT actually acts as a selector of step duration (Figure 1), which leads to the use of the follow- ing formula to evaluate the $\mathrm{WS}_{\mathrm{e}}: \mathrm{WS}_{\mathrm{e}}=\mathrm{WS}_{\mathrm{s}} \times \mathrm{SL} / 0.48$ (SL in meters) [19]. This step cadence can range from 0 to 70 steps/minute, allowing for a maximum $\mathrm{WS}_{\mathrm{e}}$ of $2 \mathrm{~km} / \mathrm{h}$ $(0.56 \mathrm{~m} / \mathrm{s})$ achievable only with $\mathrm{SL}=0.48 \mathrm{~m}$. In this article, unless otherwise defined, WS indicates $\mathrm{WS}_{\mathrm{e}}$, expressed in kilometers per hour as on the GT control panel, and the corresponding value in meters per second is also given in parentheses. Furthermore, by dividing the distance virtually covered during the session $\left(n_{\text {strides }} \times \mathrm{SL} \times 2\right.$, where $n_{\text {strides }}=$ number of strides performed) by the session duration, we could calculate mean WS during the entire GT session $\left(\mathrm{WS}_{\text {mean }}\right)$. This parameter, when compared with $\mathrm{WS}_{\mathrm{e}}$, can be helpful in understanding the effects of transitory and rest phases.

\section{Protocol 1: Retrospective Study}

We retrospectively analyzed parameter values recorded in GT sessions of 20 patients with subacute 
stroke (mean age \pm standard deviation [SD]: $59 \pm 17$ years, 5 females). At admission, 18 patients had an FAC score = 0 and 2 had an FAC score $=1$; their mean Rivermead Mobility Index was $1.8 \pm 1.1$. Their rehabilitation program consisted of two conventional physiotherapy sessions a day for 2 or 3 months. Starting about 1.5 months after the stroke event, one daily session was replaced by a GT session for 4 consecutive weeks. At the first GT session, seven patients (age: $63 \pm 11$ years) had an FAC score $=0$ (no functional ambulation); seven (age: $68 \pm$ 6 years) had an FAC score $=1$ (requiring another person to support their body weight while walking); and six (age: $46 \pm 22$ years) had an FAC score $\geq 2$ (4 with an FAC score $=2$ [i.e., requiring slight manual contact], and 2 with an FAC score $=3$ [i.e., requiring supervision during walking]). For each session, the $\mathrm{WS}_{\mathrm{s}}$ and $\mathrm{BWS}_{\mathrm{s}}$ were selected according to the patient's motor ability, as visually assessed by the physiotherapist. BWS $_{\mathrm{s}}$ was selected with the two plate anterior borders aligned (see next section, "Protocol 2: Initial Position," for details). These values could be modified during the first part of the session if judged inappropriate. SL was selected primarily in accordance with the values usually achieved by patients with chronic stroke [20] and patient stature and was therefore quite fixed among patients and sessions (SL $=39 \pm 2 \mathrm{~cm}$ ). Thus, we did not analyze it further. We should note that the choice of an SL lower than the maximum possible $(48 \mathrm{~cm})$ implied a $\mathrm{WS}_{\mathrm{e}}$ lower than the device's maximum because of the limit on step cadence mentioned earlier.

During the first GT session, $\mathrm{BWS}_{\mathrm{s}}$ was selected to be about 40 to 60 percent and $\mathrm{WS}_{\mathrm{s}}$ about 1.2 to $1.8 \mathrm{~km} / \mathrm{h}$, corresponding to a $\mathrm{WS}_{\mathrm{e}}$ of $1.0-1.5 \mathrm{~km} / \mathrm{h}(0.28-0.42 \mathrm{~m} / \mathrm{s})$, according to each patient's capacity. The $\mathrm{BWS}_{\mathrm{s}}$ was reduced as soon as the patient could take his/her full weight, and the $\mathrm{WS}_{\mathrm{s}}$ was increased as soon as the patient could perform the task without overstraining and/or experiencing discomfort [5]. The physiotherapist involved in the GT treatment prepared the patients, chose the parameters, provided verbal feedback to the patient during the GT session, and manually controlled the patients' paretic knee, if needed.

During each session, the physiotherapist recorded the following parameters: session duration (maximum: $20 \mathrm{~min}$ ); the number of strides performed (clearly depending on session duration and $\mathrm{WS}_{\mathrm{e}}$ ); heart rate (HR) increment $\left(\Delta \mathrm{HR}=\mathrm{HR}_{\text {post }}-\mathrm{HR}_{\text {pre }}\right.$, where $\Delta \mathrm{HR}=\mathrm{HR}$ increment, $\mathrm{HR}_{\text {post }}=\mathrm{HR}$ after the session, and $\mathrm{HR}_{\text {pre }}=\mathrm{HR}$ before the session); its percentage relative to maximum safe increment of cardiovascular rate $\left(c=\Delta \mathrm{HR} /\left(\mathrm{HR}_{\mathrm{max}}-\mathrm{HR}_{\mathrm{pre}}\right) \times\right.$
100 , where $c$ = percentage of maximum safe increment of cardiovascular rate and $\mathrm{HR}_{\max }=190$ - age [21]); and the FAC score of the patient.

\section{Protocol 2: Initial Position}

The $\mathrm{BWS}_{\mathrm{s}}$ and $\mathrm{BWS}_{\mathrm{e}}$ values were measured in static and training conditions, respectively, with the GT dynamometer (Figure 1), with a sampling frequency of about $3 \mathrm{~Hz}$. BWS sas 20 percent of the body weight of each subject (corresponding to a vertical force of $161 \pm 27 \mathrm{~N}$ ), and it was measured in three different initial platform positions: position $\mathrm{A}$, at maximum sagittal distance $(0.48 \mathrm{~cm})$; position B, with anterior borders $24 \mathrm{~cm}$ apart; and position $\mathrm{C}$, with anterior borders aligned. The measurements were recorded twice with the left and right limb forward (positions $\mathrm{A}$ and $\mathrm{B}$ ) and in the standing position (position C). Then, $\mathrm{BWS}_{\mathrm{e}}$ was recorded during 20 steps performed by eight nondisabled subjects (mean age: $34 \pm$ 4 years; mean body mass: $82 \pm 14 \mathrm{~kg}$ ) in the middle of six brief GT sessions. For all eight subjects, WS was $2 \mathrm{~km} / \mathrm{h}$ $(0.56 \mathrm{~m} / \mathrm{s})$ and $\mathrm{SL}$ was $0.48 \mathrm{~cm}$. At the same time, the accelerations of the apex of the patient's harness (the horizontal bar to which the harness clamps were fixed [Figure 1]) were measured with a triaxial accelerometer (Vibracting ${ }^{\circledR}$, Sensorize s.r.l.; Rome, Italy) with a sampling frequency of $100 \mathrm{~Hz}$. The root-mean-square (RMS) of acceleration measured in the anteroposterior (AP), laterolateral (LL), and craniocaudal (CC) directions was evaluated.

\section{Protocol 3: Effective BWS for Patients}

The $\mathrm{BWS}_{\mathrm{e}}$ and acceleration RMS were also recorded for seven patients with hemiplegia due to subacute stroke (mean age: $64 \pm 7$ years, mean body mass: $71 \pm 12 \mathrm{~kg}$ ) during an early GT session. For them, we recorded BWS in position $C$ with the nonparetic limb extended. We normalized the measured values to account for differences in WS and SL according to this formula: $\mathrm{RMS}_{\mathrm{n}}=\mathrm{RMS} /$ $\mathrm{WS}^{2} \times \mathrm{SL}$, where $\mathrm{RMS}_{\mathrm{n}}=$ normalized RMS [22]. We compared these normalized values with the relevant values of nondisabled subjects recorded at position C. All participants could request that the sessions be stopped at any time so they could rest.

\section{Statistical Analysis}

Mean \pm SD was computed for each of the recorded parameters. We used repeated measures analyses of variance (ANOVAs) to assess the effects of session repetition (within factor) and the FAC score recorded at the first GT session (fixed factor, three levels: 0,1 , or $\geq 2$ ) on $\mathrm{BWS}_{\mathrm{s}}$, 
$\mathrm{WS}_{\mathrm{e}}$, number of strides performed, and $\Delta \mathrm{HR}$ (dependent variables). Because not all patients performed 20 GT sessions, we conducted this ANOVA on the first 15 GT sessions, which 18 of the 20 patients completed. These ANOVA calculations were followed by post hoc analyses with the Tukey test.

We used an exponential function $\left(y=a+b \cdot e^{s / \tau}\right.$, where $y=$ the function approximating the investigated parameter at which the fit is applied; the parameters of the exponential fit are $a=$ constant, $b=$ coefficient, $e=$ Eulerian number, $\tau=$ constant time, and $s=$ the number of the GT session) to fit the parameter values recorded during progressive GT session. We used coefficient of determination $R^{2}$ to assess the goodness of this fit. We computed Pearson $(R)$ and Spearman $(\rho)$ coefficients to evaluate correlations among recorded values for continuous and ordinal measurements, respectively.

We performed linear regression to assess the correlation between $\mathrm{BWS}_{\mathrm{e}}$ and the position at which $\mathrm{BWS}_{\mathrm{s}}$ was selected in protocol 2. Finally, in protocol 3, we used $t$ tests to compare patients and nondisabled subjects in both mean $\mathrm{BWS}_{\mathrm{e}}$ and acceleration RMS.

\section{RESULTS}

\section{Protocol 1: Retrospective Study}

The first GT session was performed $48 \pm 18$ days after the stroke event. The 20 patients performed 342 of the 400 planned GT sessions (85\%), with $17 \pm 3$ sessions per patient. Only 6 subjects performed all 20 prescribed sessions. In accordance with the defined protocol (see "Methods" section), at the first session the mean value of BWS $_{\mathrm{S}}$ was $52.6 \pm 15.7$ percent of the patient's body weight and the mean $\mathrm{WS}_{\mathrm{s}}$ was $1.3 \pm 0.2 \mathrm{~km} / \mathrm{h}(0.35 \pm 0.05 \mathrm{~m} / \mathrm{s})$.

Repeated measures ANOVA (performed on the first 15 GT sessions) showed that the number of the session significantly affected $\mathrm{BWS}_{\mathrm{S}}\left(F_{2,14}=45.65, p<0.001\right)$, $\mathrm{WS}_{\mathrm{e}}\left(F_{2,14}=17.15, p<0.001\right)$, and number of strides performed $\left(F_{2,14}=12.95, p=0.001\right)$, but not $\Delta \operatorname{HR}\left(F_{2,14}=\right.$ $0.74, p=0.732)$. The FAC score recorded at the first GT session significantly affected $\mathrm{BWS}_{\mathrm{S}}\left(F_{2,14}=10.32, p=\right.$ $0.002)$, but not $\mathrm{WS}_{\mathrm{e}}\left(F_{2,14}=0.348, p=0.712\right)$, number of strides performed $\left(F_{2,14}=0.53, p=0.600\right)$, or $\Delta \operatorname{HR}\left(F_{2,14}=\right.$ $0.908, p=0.424)$. Post hoc analyses showed significant differences in $\mathrm{BWS}_{\mathrm{s}}$ for patients with an FAC score $=0$ compared with patients with an FAC score $=1$ and $\geq 2$ ( $p \leq 0.005$ each), but not between the latter two groups $(p=0.99)$.
Figure 2 shows the progressive reduction of $\mathrm{BWS}_{\mathrm{s}}$ and the progressive increment of $\mathrm{WS}_{\mathrm{e}}$ averaged among patients grouped by FAC score recorded at the first GT session. Because not all 400 sessions were performed, the values for the last sessions were not computed for all patients. All parameters except $\Delta \mathrm{HR}$ were well fitted by an exponential function (mean $R^{2}=0.83 \pm 0.16$ ).

Patients with an initial FAC score $=0$ showed low $\tau$ values for number of strides performed ( $\tau=1.6$ sessions), WS ( $\tau=2)$, and FAC score $(\tau=8.6)$, indicating quick variations in these parameters. However, these patients also showed the slowest $\mathrm{BWS}_{\mathrm{S}}$ decrement $(\tau=67.5)$, whereas a quick decrement of $\mathrm{BWS}_{\mathrm{S}}$ (corresponding to lower values of $\tau$ ) was recorded for patients with an FAC score $=1(\tau=10.3)$ or $\geq 2(\tau=6.6)$, despite the similar BWS $_{\mathrm{S}}$ selected for all patients at the first GT session.

For patients with an FAC score $\geq 2$ at the first GT session, the increment of performed strides ( $\tau=14.4$ sessions), WS ( $\tau=16.8)$, and overall FAC score $(\tau=73.1)$ were slow and quite linear. This slow, linear increment occurred despite the patients' fast decrement of $\mathrm{BWS}_{\mathrm{s}}$ and their capacity to complete the 20-minute sessions, even at the second session. Furthermore, the asymptote of the exponential fit for their WS was $2.2 \mathrm{~km} / \mathrm{h}(0.61 \mathrm{~m} /$ $\mathrm{s}$ ), over the limit of the selectable WS, which was $2 \mathrm{~km} / \mathrm{h}$ $(0.56 \mathrm{~m} / \mathrm{s})$ for $\mathrm{SL}=0.48 \mathrm{~cm}$ and $1.7 \mathrm{~km} / \mathrm{h}(0.47 \mathrm{~m} / \mathrm{s})$ for $\mathrm{SL}=0.40 \mathrm{~cm}$.

The $\mathrm{WS}_{\text {mean }}$ computed over all 342 sessions was 18 percent less than $\mathrm{WS}_{\mathrm{e}}$ among all patients. Moreover, these two parameters were greatly but not perfectly related $(R=0.87)$.

The $\Delta$ HR did not vary greatly among sessions, with a mean of $16.8 \pm 15.7$ beats per minute (bpm) (higher for patients with higher initial FAC scores [Figure 2]). The mean $c$ was $18.3 \pm 8.0$ percent. The $\Delta \mathrm{HR}$ was not significantly related to $\mathrm{BWS}_{\mathrm{S}}(R=-0.04, p=0.42), \mathrm{WS}_{\mathrm{e}}(R=$ $0.07, p=0.22)$, the number of the session $(R=-0.02, p=$ $0.78)$, or the FAC score ( $\rho=0.06, p=0.27)$; however, it was slightly but significantly correlated only with the number of strides performed $(R=0.14, p=0.01)$.

At dismissal, these 20 patients had a mean FAC score of $3.9 \pm 1.0$ and a mean Rivermead Mobility Index of $8.8 \pm$ 3.5 , and they were able to walk a mean $161 \pm 83 \mathrm{~m}$ in $6 \mathrm{~min}$. This performance corresponds to a $\mathrm{WS}_{\text {mean }}$ of $1.61 \pm$ $0.83 \mathrm{~km} / \mathrm{h}(0.45 \pm 0.23 \mathrm{~m} / \mathrm{s})$. These values were similar for all three subgroups of patients with an FAC score of 0,1 , and $\geq 2$ recorded at the first GT session. Some correlations were found between the values of selected parameters in the last GT session and clinical scores at dismissal. 


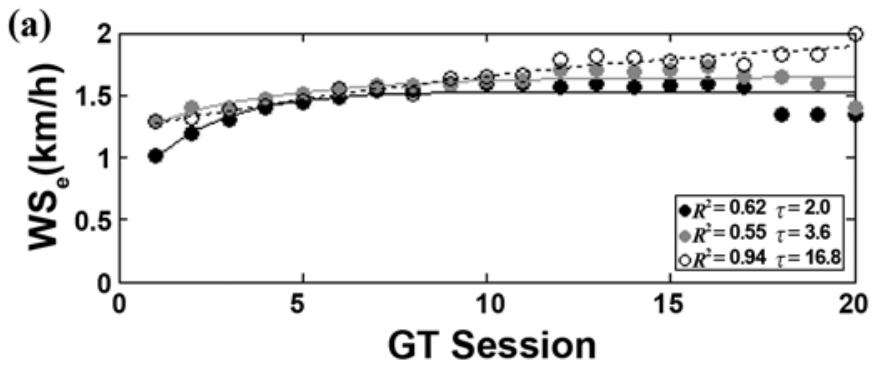

(b)
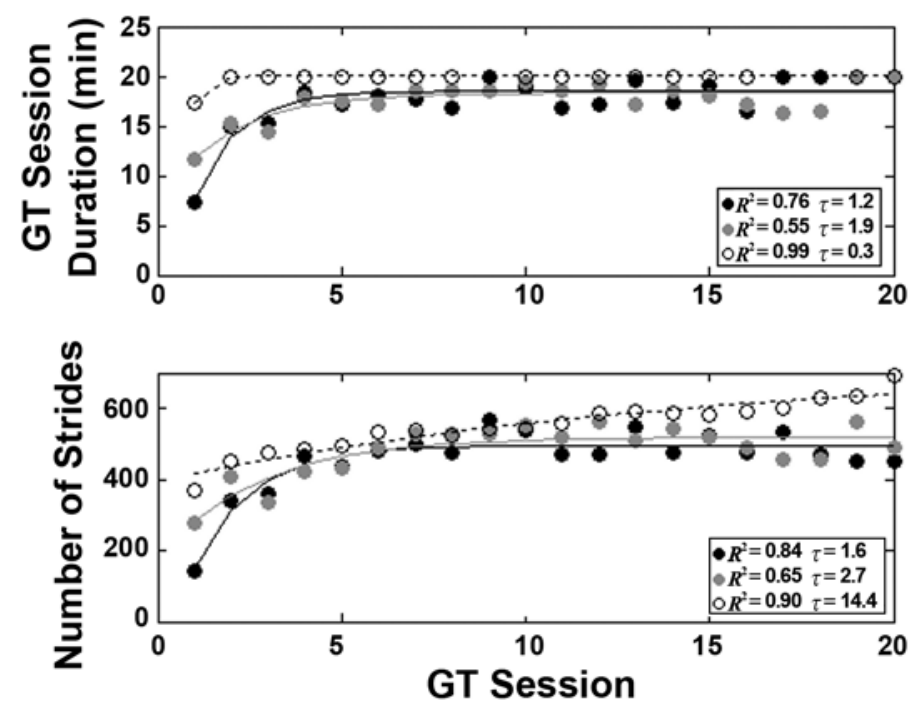
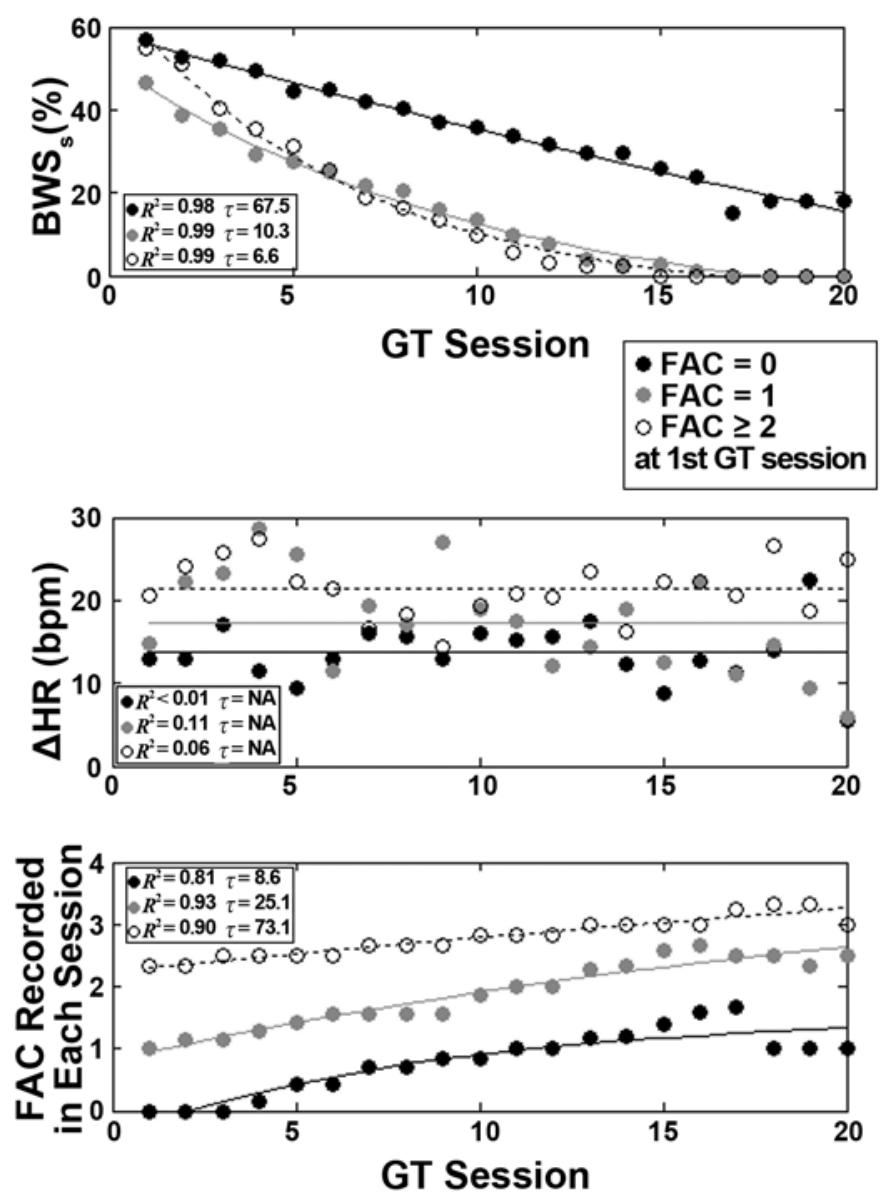

Figure 2.

Mean parameter values along 20 Gait Trainer $($ GT) sessions for patients (black circles: initial Functional Ambulation Category $($ FAC) score = 0; gray circles: FAC score = 1; empty circles: FAC score $\geq 2$ ) and relevant exponential fits (black, gray, and dotted lines, respectively). (a) Selected parameter values, effective walking speed $\left(\mathrm{WS}_{\mathrm{e}}\right)$ and body-weight support in a static posture $\left(\mathrm{BWS}_{\mathrm{S}}\right)$. (b) Values of measured parameters (duration of session, heart rate increment $(\Delta \mathrm{HR})$, number of strides per session, FAC score recorded at each session). Values of $R^{2}$ (coefficient of determination measuring the goodness of the fit) and $\tau$ (constant time of the exponential fit) were reported for each group and each parameter. For $\Delta$ HR, exponential fit failed to represent values $\left(R^{2}<0.1\right)$ and for this parameter, mean line for each group was plotted.

The Rivermead Mobility Index related to the last $\mathrm{WS}_{\mathrm{e}}(\rho=$ $0.54, p=0.02)$ and $\mathrm{BWS}_{\mathrm{s}}(\rho=-0.45, p=0.048)$, whereas the FAC score related only to $\operatorname{BWS}_{\mathrm{S}}(\rho=-0.55, p=$ $0.01)$. The distance covered in $6 \mathrm{~min}$, even if related to the Rivermead Mobility Index ( $\rho=0.60, p=0.01$ ), was poorly and not significantly correlated with the parameter values recorded at the last GT session: $\mathrm{BWS}_{\mathrm{S}}(R=-0.32$, $p=0.21), \mathrm{WS}_{\mathrm{e}}(R=0.27, p=0.29)$, and number of strides performed $(R=0.19, p=0.46)$.

\section{Protocol 2: Initial Position}

The mean values of the differences between $\mathrm{BWS}_{\mathrm{e}}$ and $\mathrm{BWS}_{\mathrm{s}}$ measured during 10 strides starting from three different initial positions are shown in Figure 3. The linear relationship between this difference and the initial plate distance can identify an optimal position for plates. This optimal position is with their anterior borders positioned $12 \mathrm{~cm}$ apart, at which mean $\mathrm{BWS}_{\mathrm{e}}$ roughly coincides with $\mathrm{BWS}_{\mathrm{s}}$. In contrast, position $\mathrm{C}$, probably the easiest to reproduce, implied an overestimation of $\mathrm{BWS}_{\mathrm{e}}$ of about 4 percent of each subject's weight.

\section{Protocol 3: Effective BWS for Patients}

The representative results of a patient with left hemiparesis are shown in Figure 4. An asymmetric pattern is evident: all the minima of $\mathrm{BWS}_{\mathrm{e}}$ corresponded to extension 


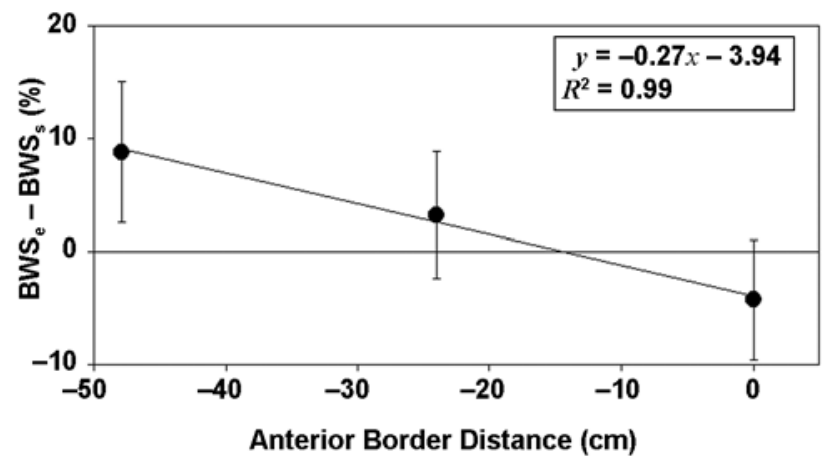

Figure 3.

Mean \pm standard deviation of difference between effective bodyweight support $\left(\mathrm{BWS}_{\mathrm{e}}\right)$ and BWS in static posture $\left(\mathrm{BWS}_{\mathrm{s}}\right)$ recorded in eight nondisabled subjects in relationship to initial distance between anterior borders of two Gait Trainer plates. Linear regression and its equation are also shown ( $x$ is the anterior border distance; $y$ is the fitting function approximating $\mathrm{BWS}_{\mathrm{s}}-\mathrm{BWS}_{\mathrm{e}}$, and the goodness of this approximation was measured by the coefficient of determination $R^{2}$.

of the nonparetic limb (i.e., its stance phase), whereas the higher maxima were close to the beginning of the paretic step, i.e., when the paretic limb accepts the load. Similar patterns were observed for acceleration, with higher peak-to-peak values for acceleration in the LL direction $\left(a_{\mathrm{LL}}\right)$ and acceleration in the AP direction $\left(a_{\mathrm{AP}}\right)$ during the step of the paretic leg (dark gray bands in Figure 4).

The mean values of the selected parameters measured for the seven patients were $\mathrm{BWS}_{\mathrm{S}}=31 \pm 11$ percent, $\mathrm{WS}_{\mathrm{e}}=$ $1.5 \pm 0.3 \mathrm{~km} / \mathrm{h}(0.42 \pm 0.08 \mathrm{~m} / \mathrm{s}), \mathrm{SL}=0.38 \pm 0.05 \mathrm{~m}$, and $\mathrm{BWS}_{\mathrm{e}}=27 \pm 16$ percent. Despite the high variability in patient data, the mean difference between $\mathrm{BWS}_{\mathrm{e}}$ and $\mathrm{BWS}_{\mathrm{s}},-4$ percent, did not differ from that of nondisabled subjects $(p=0.94)$. Although the normalized acceleration RMS was higher in patients than in nondisabled subjects (Figure 5), this difference was significant only in the AP direction $(p=0.03)$, not in the LL $(p=0.23)$ and CC ( $p=$ $0.06)$ directions.

\section{DISCUSSION}

This study provides a systematic documentation of selectable GT parameter values, measured parameters, and their relationships. Similar to a previous study on the selection of parameters for treadmill training [18], this study on the GT, a device used for electromechanically assisted gait training, pays particular attention to WS, harness oscillations, and BWS. Moreover, it introduces and quantifies the difference between selected and effective parameter values, which is usually omitted in GT studies.

The results obtained for FAC score agreed with previously reported results on the effectiveness of robotic devices for gait recovery [10]. We progressively reduced BWS $_{\mathrm{S}}$ in successive GT sessions: slowly (high value of $\tau$ ) for the most-affected patients (initial FAC score $=0$ ), and quickly for the less-affected patients (FAC score $\geq 2$ ), despite similar values recorded at the first GT session among all patients. Notably, the $\mathrm{BWS}_{\mathrm{s}}$ selected in the first GT session was about 45 to 60 percent of body weight; that is higher than the 30 percent previously described [23]. The GT Handbook suggested a maximum BWS of 35 percent for nonambulatory patients with hemiparesis and a maximum of 10 percent in patients walking with aid, including support [19]. Furthermore, clinical studies have recommended BWS limits of 30 percent on the treadmill [12] and 40 percent on the Lokomat ${ }^{\circledR}$ [23] for maintaining the activity of antigravitational muscles. In contrast, a review on treadmill parameter selection stated that adequate support was 35 to 50 percent [18]. Ivanenko et al. have shown that lowerlimb kinematics can be accurately controlled over a wide range of BWS and WS. Despite obvious changes in limb kinetics recorded for very high BWS, shown by recorded reaction forces and muscle activity, the authors stated that a high level of BWS may help severely affected patients with spinal cord injuries [24]. Our results suggest that this approach can be extended to patients with subacute stroke. In fact, the high BWS values observed in our study allowed the patients to begin performing task-oriented, intensive exercises without joint overloading [25].

These high $\mathrm{BWS}_{\mathrm{s}}$ values observed during the first GT session in all patients could be left to the discretion of physiotherapists in the first training sessions. The value probably depends also on a patient's need for a familiarization period with the GT task that induces gait-like movements, simulating stance and swing phases [26]. For the patients with an FAC score $\geq 1$, about five GT sessions were needed before recommended BWS levels were assumed (about 30\%). Conversely, we recorded a slow reduction of $\mathrm{BWS}_{\mathrm{s}}$ and a fast increment of $\mathrm{WS}_{\mathrm{e}}$ for the most-affected patients (FAC score $=0$ ), which presumably indicates physiotherapists' preference for intensive training on kinematics (WS and number of strides performed) more than kinetics $\left(\mathrm{BWS}_{\mathrm{s}}\right)$. Our results showed 


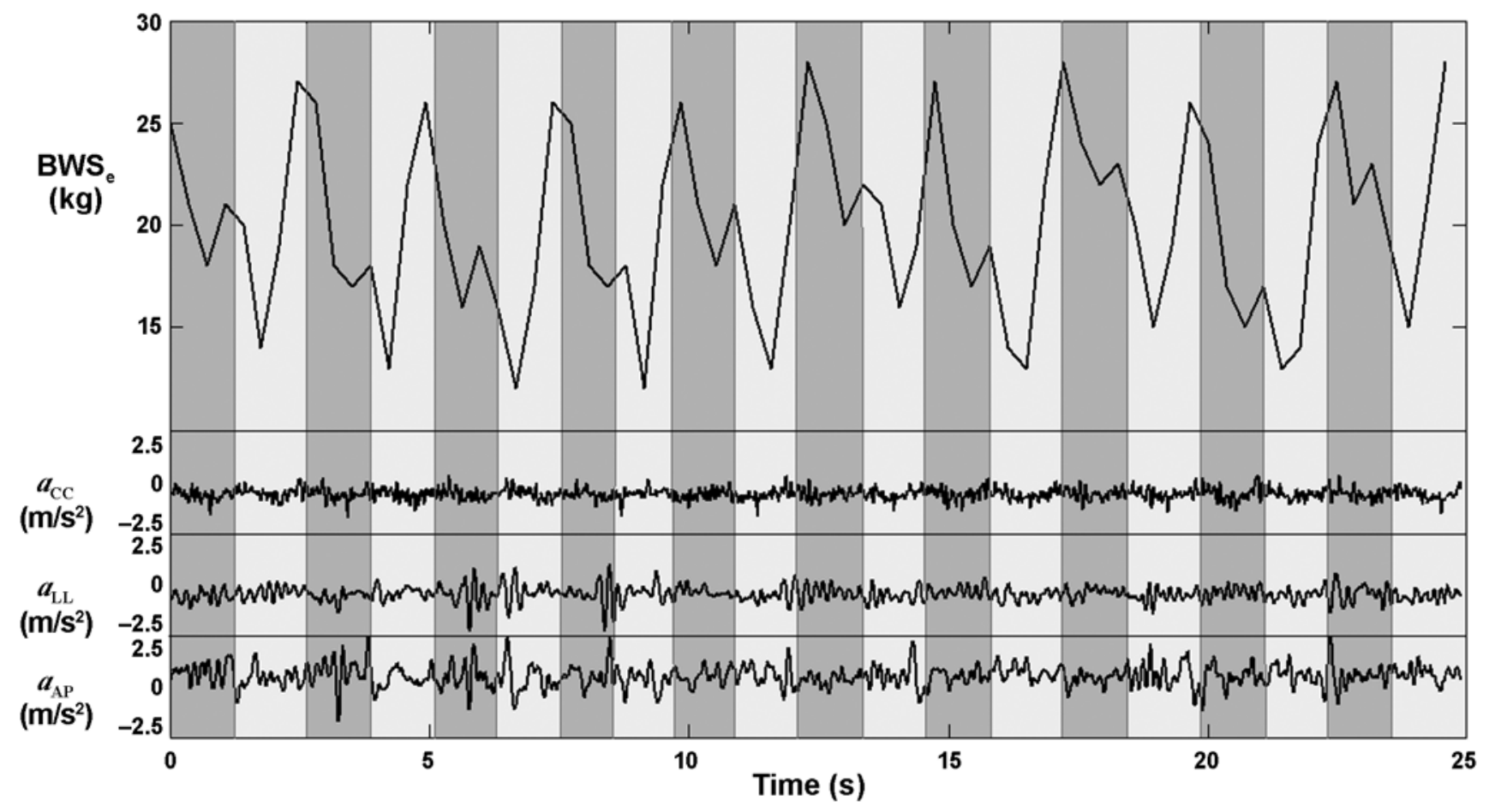

Figure 4.

Top graph shows effective body-weight support $\left(\mathrm{BWS}_{\mathrm{e}}\right.$ ) recorded during 10 strides for representative patient (age $=66$ years, body mass $=78 \mathrm{~kg}$, Functional Ambulation Category score $=0$ ). Bottom graphs show relevant acceleration $(a)$ in craniocaudal $\left(a_{\mathrm{CC}}\right)$, laterolateral $\left(a_{\mathrm{LL}}\right)$, and anteroposterior $\left(a_{\mathrm{AP}}\right)$ directions. Gait Trainer parameters during this session were body-weight support in static posture $\left(\mathrm{BWS}_{\mathrm{S}}\right)=30 \mathrm{~kg}$, effective walking speed $=0.5 \mathrm{~m} / \mathrm{s}$, and step length $=0.4$. Dark and light gray bands represent steps of paretic and nonparetic limbs, respectively.

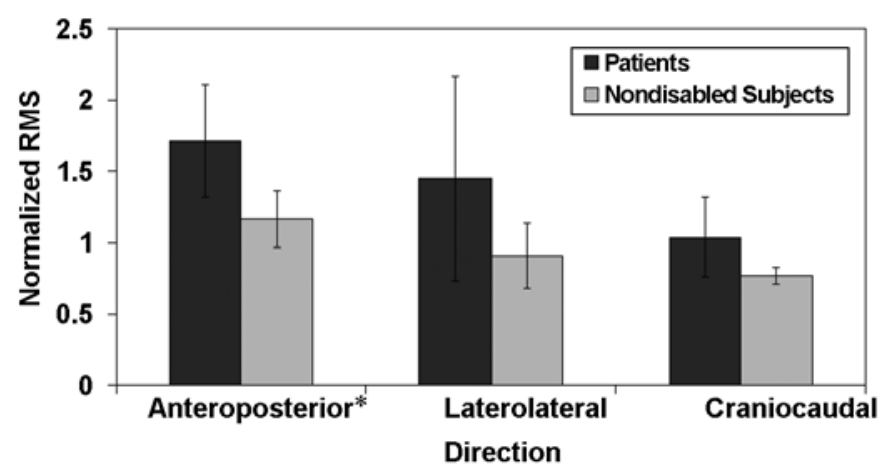

Figure 5.

Mean \pm standard deviation acceleration normalized root-mean-square (RMS) for patients (dark gray) and nondisabled subjects (light gray) evaluated in anteroposterior, laterolateral, and craniocaudal directions. $\stackrel{*}{p}<0.05$.

that this preference resulted in patients performing training under safe cardiovascular conditions.

Protocol 2 showed the effects of different positions of measuring $\mathrm{BWS}_{\mathrm{s}}$ on $\mathrm{BWS}_{\mathrm{e}}$. Although the position at which the anterior borders of the plates are aligned resulted in an overestimation of $\mathrm{BWS}_{\mathrm{e}}$, that position was the easiest to reproduce during the GT sessions. Furthermore, this position allows the patient to extend one limb, the less-affected limb, to support his/her body weight. During the GT session, if the $\mathrm{BWS}_{\mathrm{e}}$ is $>4$ percent lower than the $\mathrm{BWS}_{\mathrm{S}}$ measured in the same position, then the patient's motor ability may have been underestimated. Conversely, if $\mathrm{BWS}_{\mathrm{e}}$ is consistently higher than $\mathrm{BWS}_{\mathrm{s}}$, the patient is not actively working and one should consider the possibility of a $\mathrm{BWS}_{\mathrm{S}}$ increment.

In protocol 3, when we further analyzed patients' performances, we found that $\mathrm{BWS}_{\mathrm{e}}$ and body accelerations varied between the two limb steps. This asymmetric pattern was due to the different capacities of the paretic and nonparetic limbs to support body weight. For this reason, continuous monitoring by the physiotherapist of $\mathrm{BWS}_{\mathrm{e}}$ during the GT session can reveal information about patients' compliance with exercise. Overload may cause hyperflexion, hyperextension, and/or a varus knee. 
Higher accelerations were observed for patients with stroke than for nondisabled subjects; this was particularly evident in the AP direction, probably because this direction is the less mechanically constrained. In fact, vertical movements are controlled by a vertical rope supporting the patient's body weight, whereas the lateral chains partially drive the LL movements (Figure 1). These results indicate that GT can roughly assist in controlling patient oscillations during therapeutic sessions.

We observed two important results related to WS. First, for less-affected patients, the WS limit of $2 \mathrm{~km} / \mathrm{h}$ $(0.55 \mathrm{~m} / \mathrm{s})$ may be too low. A recent study based on accelerometric measurement has also shown that walking at low speed can provide altered proprioceptive inputs [27]. Second, we found that $\mathrm{WS}_{\text {mean }}$ was about 20 percent lower than $\mathrm{WS}_{\mathrm{e}}$, indicating that transitory and rest phases are not negligible and should be considered when the $\mathrm{WS}_{\mathrm{e}}$ for the next session is selected.

The number of strides performed by our patients during the 20 min GT session was similar to that previously reported by Pohl et al. (mean 425 strides during the first 2 weeks of therapy and 538 during the following 2 weeks) [3]. This number is higher than that reported in a previous study on the number of strides performed during over-ground walking by patients manually supported by physiotherapists (about 100 strides) [28]. Four hundred strides, with a mean SL of $0.39 \mathrm{~m}$ (i.e., stride length of $0.78 \mathrm{~m}$ ) corresponds to a covered distance of $312 \mathrm{~m}$ performed by all our patients in $<20 \mathrm{~min}$. So, the $\mathrm{WS}_{\text {mean }}$ during a GT session was about $0.94 \mathrm{~km} / \mathrm{h}$, with peaks of $\mathrm{WS}_{\mathrm{e}}$ of about $1.6 \mathrm{~km} / \mathrm{h}$. Similar mean velocity $(0.84 \mathrm{~km} / \mathrm{h})$ was reported by Mehrholz et al. during the 6-minute walk test (6MWT) for the less-affected patients only (FAC score $=2.0 \pm 1.5$ ) [29]. Conversely, the $\mathrm{WS}_{\text {mean }}$ achieved during GT training by our patients was much higher than the over-ground velocities recorded in the most-affected patients in the Mehrholz et al. study $(0.16 \mathrm{~km} / \mathrm{h}$ for patients with an FAC score $=0.4 \pm 0.7$ and $0.51 \mathrm{~km} / \mathrm{h}$ for an FAC score $=1.2 \pm 1.3$ ) [29]. At dismissal, our patients performed the 6MWT with a $\mathrm{WS}_{\text {mean }}$ similar to the maximum achieved on the GT (about $1.6 \mathrm{~km} / \mathrm{h}$ ).

The patient's locomotor abilities at dismissal were found to be only partially related to the $\mathrm{BWS}_{\mathrm{s}}$ and $\mathrm{WS}_{\mathrm{e}}$ recorded in the last GT session. This limited relationship was probably due to all the patients' GT parameter values converging to the ideal values at the end of the GT training $\left(\mathrm{BWS}_{\mathrm{s}}=0 \%, \mathrm{WS}_{\mathrm{e}}=2 \mathrm{~km} / \mathrm{h}\right.$, number of strides performed $=700=35$ strides $/ \mathrm{min}$ in $20 \mathrm{~min}$ ).
Moreover, by measuring the $\Delta \mathrm{HR}$, we noticed that the patients performed all these steps under safe cardiovascular conditions. In fact, the $\Delta \mathrm{HR}$ was about $20 \mathrm{bpm}$ and poorly correlated with number of strides performed.

In light of these results, one could conceivably hypothesize that the limit on the selectable WS (due to the limit on SL) could restrain the functional walking patterns in the less-affected patients. This limit of the machine, therefore, could contrast with the physiotherapist's choice of an intensive kinematic training. For these patients, the GT can be helpful to guarantee safe conditions in the first part of their gait-oriented rehabilitative program. But then, the over-ground walking training is presumably more ecological and appropriate because it allows a more functional gait.

The main limitation of this study is the retrospective design of protocol 1. And, in fact, a rigorous analysis of the relationship of patients' over-ground walking performance with the values of selected GT parameters is lacking in this study and should be investigated in further studies. Another limit is the small sample size of protocols 2 and 3. Future clinical trials should be specifically designed to provide guidance on which parameters to select in specific clinical situations. Also, they should include control groups receiving only conventional therapy. Despite the interesting results of this study, further investigations would be required to highlight the effects of parameter selection on joint kinematics. Furthermore, other parameters should be considered, including SL, physiotherapist manual assistance, handrail hold, and frequency of GT sessions per week.

\section{CONCLUSIONS}

Our results provide a rational framework for selecting GT parameter values, although additional studies are needed. These studies could improve the effectiveness of electromechanically assisted gait training devices based on effective choices of their kinematic and kinetic parameters. The main clinical findings of this study are (1) early, safe, and kinematically intensive training can require the use of high BWS, i.e., a low kinetic demand; (2) for the less-affected patients, intensive training can be restrained by the limit of $2 \mathrm{~km} / \mathrm{h}$ for the GT WS, implying the need for another form of intervention; (3) $\mathrm{BWS}_{\mathrm{s}}$ should be selected with the two plates aligned; and (4) $\mathrm{BWS}_{\mathrm{e}}$ and the width of patients' sways during the GT session should 
be monitored and the initially selected parameters should be changed where necessary.

\section{ACKNOWLEDGMENTS}

\author{
Author Contributions: \\ Study concept and design: M. Bragoni, G. Morone. \\ Acquisition of data: G. Morone. \\ Analysis and interpretation of data: M. Iosa. \\ Drafting of manuscript: M. Iosa. \\ Critical revision of manuscript for important intellectual content: \\ D. De Angelis, V. Venturiero, P. Coiro, L. Pratesi. \\ Statistical analysis: M. Iosa. \\ Obtained funding: S. Paolucci. \\ Study coordination and supervision: S. Paolucci.
}

Financial Disclosures: The authors have declared that no competing interests exist.

Funding/Support: This material was based on work supported by the Italian Ministry of Health (grant 2009G41) and by the Fondazione Santa Lucia, Istituto di Ricerca e Cura a Carattere Scientifico [Santa Lucia Foundation, Scientific Institute for Research Hospitalization and Health Care], Rome, Italy.

Institutional Review: This study was approved by the ethical committee of the Fondazione Santa Lucia, Istituto di Ricerca e Cura a Carattere Scientifico [Santa Lucia Foundation, Scientific Institute for Research Hospitalization and Health Care], Rome, Italy, and informed consent was obtained from each participant.

Participant Follow-Up: The authors are considering a follow-up assessment to this study, at which time they would contact the current participants and communicate the results of this study.

\section{REFERENCES}

1. Hesse S, Sarkodie-Gyan T, Uhlenbrock D. Development of an advanced mechanised gait trainer, controlling movement of the centre of mass, for restoring gait in non-ambulant subjects. Biomed Tech (Berl). 1999;44:194-201.

[PMID: 10472726]

DOI:10.1515/bmte.1999.44.7-8.194

2. Hesse S, Uhlenbrock D, Werner C, Bardeleben A. A mechanized gait trainer for restoring gait in nonambulatory subjects. Arch Phys Med Rehabil. 2000; 81(9):1158-61.

[PMID: 10987154]

DOI:10.1053/apmr.2000.6280

3. Pohl M, Werner C, Holzgraefe M, Kroczek G, Mehrholz J, Wingendorf I, Hoölig G, Koch R, Hesse S. Repetitive locomotor training and physiotherapy improve walking and basic activities of daily living after stroke: A single-blind, randomized multicentre trial (DEutsche GAngtrainerStudie, DEGAS). Clin Rehabil. 2007;21(1):17-27.

[PMID: 17213237]

DOI:10.1177/0269215506071281
4. Tong RK, Ng MF, Li LS. Effectiveness of gait training using an electromechanical gait trainer, with and without functional electric stimulation, in subacute stroke: A randomized controlled trial. Arch Phys Med Rehabil. 2006; 87(10):1298-1304. [PMID: 17023237]

DOI:10.1016/j.apmr.2006.06.016

5. Werner C, Von Frankenberg S, Treig T, Konrad M, Hesse $\mathrm{S}$. Treadmill training with partial body weight support and an electromechanical gait trainer for restoration of gait in subacute stroke patients. A randomized crossover study. Stroke. 2002;33(12):2895-2901. [PMID: 12468788$]$ DOI:10.1161/01.STR.0000035734.61539.F6

6. Peurala SH, Tarkka IM, Pitkänen K, Sivenius J. The effectiveness of body weight-supported gait training and floor walking in patients with chronic stroke. Arch Phys Med Rehabil. 2005;86(8):1557-64.[PMID: 16084808] DOI:10.1016/j.apmr.2005.02.005

7. Dias D, Laíns J, Pereira A, Nunes R, Caldas J, Amaral C, Pires S, Costa A, Alves P, Moreira M, Garrido N, Loureiro L. Can we improve gait skills in chronic hemiplegics? A randomised control trial with gait trainer. Eur Medicophys. 2007;43(4):499-504. [PMID: 18084173]

8. Hidler J, Nichols D, Pelliccio M, Brady K, Campbell DD, Kahn JH, Hornby TG. Multicenter randomized clinical trial evaluating the effectiveness of the Lokomat in subacute stroke. Neurorehabil Neural Repair 2009;23(1):5-13.

[PMID: 19109447]

DOI:10.1177/1545968308326632

9. Peurala SH, Airaksinen O, Huuskonen P, Jäkälä M, Juhakoski M, Sandell K, Tarkka IM, Sivenius J. Effects of intensive therapy using gait trainer or floor walking exercises early after stroke. J Rehabil Med. 2009;41(3):166-73. [PMID: 19229450] DOI:10.2340/16501977-0304

10. Mehrholz J, Werner C, Kugler J, Pohl M. Electromechanical-assisted training for walking after stroke. Cochrane Database Syst Rev. 2007;4:CD006185._PMID: 17943893]

11. Hesse S, Werner C, Paul T, Bardeleben A, Chaler J. Influence of walking speed on lower limb muscle activity and energy consumption during treadmill walking of hemiparetic patients. Arch Phys Med Rehabil.2001;82(11):1547-50. [PMID: 11689974] DOI:10.1053/apmr.2001.26607

12. Hesse S, Helm B, Krajnik J, Gregoric M, Mauritz KH. Treadmill training with partial body weight support: Influence of body weight release on the gait of hemiparetic patients. J Neuro Rehabil. 1997;11(1):15-20.

DOI:10.1177/154596839701100103

13. Moseley AM, Stark A, Cameron ID, Pollock A. Treadmill training and body-weight support for walking after stroke. Stroke. 2003;34:3006. DOI:10.1161/01.STR.0000102415.43108.66 
14. Dobkin BH. Strategies for stroke rehabilitation. Lancet Neurol. 2004;3(9):528-36. [PMID: 15324721]

DOI:10.1016/S1474-4422(04)00851-8

15. McCain KJ, Pollo FE, Baum BS, Coleman SC, Baker S, Smith PS. Locomotor treadmill training with partial bodyweight support before overground gait in adults with acute stroke: A pilot study. Arch Phys Med Rehabil. 2008; 89(4):684-91. [PMID: 18373999]

DOI:10.1016/j.apmr.2007.09.050

16. Colombo G. The "Lokomat"-A driven ambulatory orthosis. Med Orth Tech. 2000;6:178-81.

17. Franz JR, Glauser M, Riley PO, Della Croce U, Newton F, Allaire PE, Kerrigan DC. Physiological modulation of gait variables by an active partial body weight support system. J Biomech. 2007;40(14):3244-50.[PMID: 17583716] DOI:10.1016/j.jbiomech.2007.04.016

18. Chen G, Patten C. Treadmill training with harness support: Selection of parameters for individuals with poststroke hemiparesis. J Rehabil Res Dev. 2006;43(4):485-98.

[PMID: 17123188] DOI:10.1682/JRRD.2005.04.0063

19. Reha-Stim. Gait Trainer GT I Handbook. Berlin (Germany); 2007.

20. Patterson SL, Rodgers MM, Macko RF, Forrester LW. Effect of treadmill exercise training on spatial and temporal gait parameters in subjects with chronic stroke: A preliminary report. J Rehabil Res Dev. 2008;45(2):221-28.

[PMID: 18566940$]$ DOI:10.1682/JRRD.2007.02.0024

21. Gobelet C, Franchignoni F. Vocational rehabilitation. New York (NY): Springer; 2006.

22. Mizuike C, Ohgi S, Morita S. Analysis of stroke patient walking dynamics using a tri-axial accelerometer. Gait Posture. 2009;30(1):60-64. [PMID: 19349181]

DOI:10.1016/j.gaitpost.2009.02.017

23. Lewek MD, Cruz TH, Moore JL, Roth HR, Dhaher YY, Hornby TG. Allowing intralimb kinematic variability during locomotor training poststroke improves kinematic consistency: A subgroup analysis from a randomized clinical trial. Phys Ther. 2009;89(8):829-39. [PMID: 19520734$]$ DOI:10.2522/ptj.20080180
24. Ivanenko YP, Grasso R, Macellari V, Lacquaniti F. Control of foot trajectory in human locomotion: Role of ground contact forces in simulated reduced gravity. J Neurophysiol. 2002;87(6):3070-89. [PMID: 12037209]

25. Bernhardt J, Dewey H, Thrift A, Donnan G. Inactive and alone: Physical activity within the first 14 days of acute stroke unit care. Stroke. 2004;35(4):1005-9.

[PMID: 14988574]

DOI:10.1161/01.STR.0000120727.40792.40

26. Hesse S, Uhlenbrock D. A mechanized gait trainer for restoration of gait. J Rehabil Res Dev. 2000;37(6):701-8. [PMID: 11321006]

27. Regnaux JP, Saremi K, Marehbian J, Bussel B, Dobkin BH. An accelerometry-based comparison of 2 robotic assistive devices for treadmill training of gait. Neurorehabil Neural Repair. 2008;22(4):348-54. [PMID: 18073325]

DOI:10.1177/1545968307310050

28. Lang CE, MacDonald JR, Gnip C. Counting repetitions: An observational study of outpatient therapy for people with hemiparesis post-stroke. J Neurol Phys Ther. 2007;31(1): 3-10. [PMID: 17419883]

29. Mehrholz J, Wagner K, Rutte K, Meissner D, Pohl M. Predictive validity and responsiveness of the functional ambulation category in hemiparetic patients after stroke. Arch Phys Med Rehabil. 2007;88(10):1314-19.

[PMID: 17908575]

DOI:10.1016/j.apmr.2007.06.764

Submitted for publication April 19, 2010. Accepted in revised form October 28, 2010.

This article and any supplementary material should be cited as follows:

Iosa M, Morone G, Bragoni M, De Angelis D, Venturiero V, Coiro P, Pratesi L, Paolucci S. Driving electromechanically assisted Gait Trainer for people with stroke. J Rehabil Res Dev. 2011;48(2):135-46.

DOI:10.1682/JRRD.2010.04.0069

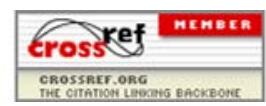


